Un chapitre concerne la taxonomie, l'origine et la domestication du lapin, un autre la Génétique formelle. La génétique de la coloration äu pelage est relativement bien connue, ce qui permet d'établir une génétique des races. Les races se différencient principalement par la coloration et les variations du pelage, la taille corporelle adulte (caractère en relation avec ceux de productivité numérique ou pondérale), la morphologie corporelle. 70 locus connus contrôlent les variations $d u$ pelage (I/3), les groupes sanguins et la production d'anticorps (I/3). D'autres contrôlent des maladies héréditaires, et de ce point de vue, le lapin est utilisé comme modèle expérimental en recherche médicale. L'on connait rarement des gènes majeurs ayant un effet sur des caractères de productivité, par contre l'on connaît des effets délétères de gènes agissant chez les homozygotes recessifs.

Un chapitre concerne la génétique des races, souches, lignées consanguines et deux autres la génétique quantitative. Les types d'expérimentations en croisement visant à caractériser les races sur le plan biologique sont indiqués. D'autres études sur ce sujet semblent nécessaires. L'on essaie de đéfinir en termes de génétique des populations, les notions de races, souches, lignées consanguines.

Une connaissance biologique du matériel animal est nécessaire pour mieux comprendre le déterminisme génétique des caractères et utiliser la variabilité pour l'amélioration. Des étuđes fondamentales, portant sur l'influence maternelle sur le poids à la naissance mise en évidence entre races de tailles adultes très différentes et sur ses interrelations avec la taille de portée, la croissance pondérale post traissance, sont discutées. De même, celles portant sur la forme de la courbe de croissance qui montrent, intra souche, des variations concernant la vitesse de croissance dans le jeune âge, le poids adulte et la vitesse d'atteinte de celui-ci. De même, celles portant sur le développement des organes et des tissus, la croissance cellulaire et biochimique. Les composantes de la fécondité des femelles semblent être maintenant relativement bien définies. Les résultats concernant l'influence de la consanguinité sur la taille de portée à la naissance sont discutés à partir des résultats bibliographiques. Les effets de la race de mâle sur cette taille de portée, mis en évidence récemment, seront à analyser plus en détail.

Les facteurs de variation génétique et non génétique des caractères quantitatifs peuvent s'analyser avec deux objectifs principaux : meilleure connaissance du déterminisme génétique des caractères, exploitation de la variabilité pour l'amélioration génétique des troupeaux. Des résultats concernant la comparaison de races acouplées en pur et en croisements sont indiqués ainsi que ceux concernant l'estimation des effets génétiques entre races et croisements. $L$ 'accent est mis, par commentaire de résultats récents à partir đe quelques exemples, sur l'intérêt d'estimer l'action conjointe des effets génétiques directs, maternels, grand maternels, sur les caractères zootechniques globaux ou leurs composantes, ainsi que l'heterosis : croisement terminal, croisement à double étage. Les résultats portant sur les paramètres génétiques (héritabilités, corrélations) des populations sont très peu nombreux. Un tableau indiquant les valeurs probables des héritabilités, pour quelques caractères, est donné. Au stade actuel, les paramètres réalisés dans le cadre d'expérimentations en sélection pourraient être recherchés.

En conclusion, l'accent est mis sur le besoin d'expérimentations en croisement et sélection pour mieux connaître les effets génétiques entre et intra populations et les exploiter en amélioration génétique, en prenant en compte le milieu.

\title{
Communications
}

\section{GENETIC ANALYSIS OF PRODUCTION TRAITS IN ITALIAN NEW ZEALAND WHITE AND CALIFORNIA PURE-BRED POPULATIONS}

\author{
E. RANDI $(*)$ and R. E. SCOSSIROLI $\left({ }^{* *}\right)$
}

(*) CUNICOOP, Via S. Donato I54, 40057 Granarolo E. (Bologna), Italy

(**) Istituto di Genetica, Università, Via F. Selmi I, 4 or26 Bologna

The preliminary results of a genetic analysis of some NZW and CAL purebred populations reared in two Cooperative Selection Centres in Emilia-Romagna (Italy) are presented. These populations are analyzed for production and survival traits.

The estimates of heritability and the phenotypic, genetic and environmental correlation coefficients are discussed. 